\title{
Microbial flora in carcinoma of oesophagus
}

\author{
IG FINLAY, PA WRIGHT, T MENZIES, CS McARDLE \\ From the Departments of Surgery and Bacteriology, Royal Infirmary, Glasgow
}

ABSTRACT The microbial flora associated with carcinoma of the oesophagus was studied in 12 patients. Oesophageal mucosa was biopsied at thoracotomy and cultured under both aerobic and anaerobic conditions. A heavy mixed growth of aerobic and anaerobic organisms was obtained in all patients. One-third of isolates were anaerobic. The flora was of oral origin. The most appropriate antibiotic combination in this study was ampicillin or penicillin with gentamicin and metronidazole.

Oesophageal resection for carcinoma is associated with a high mortality. ${ }^{1}$ At least $50 \%$ of the deaths are attributable to anastomotic leakage and associated infection. ${ }^{2}$ Despite the importance of sepsis in this situation, the microbial flora associated with carcinoma of oesophagus has not been previously investigated.

The aim of the present study, therefore, was to identify the flora associated with malignant strictures of the oesophagus and to establish the corresponding antibiotic sensitivities.

\section{Patients and methods}

Twelve consecutive patients undergoing oesophageal resection for carcinoma were included in the study. All patients had dysphagia (mean duration three months) and evidence of weight loss (mean $16 \mathrm{~kg}$ ) before surgery. Seven patients had an adenocarcinoma. Five patients with squamous carcinomas received preoperative radiotherapy. A two-part "Ivor Lewis" oesophagectomy was performed on all patients with a gastro-oesophageal anastomosis fashioned in the thorax.

At thoracotomy, specimens of oesophageal mucosa were obtained from the proximal resection line after excision of the tumour. This precluded the possibility of contamination of specimens by oral secretions. These samples were immediately transported to the bacteriology laboratory in sterile containers and also in Robertson's cooked meat medium (RCM).

Agar plates were inoculated with portions of intact and ground mucosa. The media used were (1) $5 \%$ horseblood agar, (2) "chocolate" blood agar, Address for reprint requests: Dr CS McArdle, Department of Surgery, Royal Infirmary, Glasgow G4 OSF.
(3) cysteine-lactose electrolyte deficient agar, (4) $5 \%$ horseblood agar containing $6 \mathrm{mg} / \mathrm{l}$ gentamicin and supplemented with yeast extract, cysteine hydrochloride, haemin and menadione, (5) McConkey agar. Plates containing media (1), (2), (3), and (5) were incubated at $37^{\circ} \mathrm{C}$ in $\mathrm{CO}_{2}$ for a minimum of three days. Plates containing media (1) and (4) were incubated anaerobically (BBL GasPak $\mathrm{H}_{2}+\mathrm{CO}_{2}$ ) at $37^{\circ} \mathrm{C}$ for a minimum of five days.

Portions of mucosa were also added to RCM and incubated at $37^{\circ} \mathrm{C}$ for five days. These were then subcultured to the above agar media and incubated anaerobically and also in $\mathrm{CO}_{2}$ for a further five days. Agar media used for anaerobic incubation were prereduced in an anaerobic jar.

After incubation the plates were carefully examined and representative examples of each colonial type were selected for further study. Organisms were identified by the application of the following standard techniques: growth in air; growth in air + $5 \% \mathrm{CO}_{2}$ or in an anaerobic environment; colonial morphology; microscopic morphology; Gram reaction; haemolysis; catalase test; coagulase test; motility. Enterobacteriaceae were identified using the API 20E test kit. Strictly anaerobic gramnegative bacilli were regarded as bacteroides. Bacteroides fragilis was further identified by antibiogram and Bacteroides melaninogenicus by the black or grey colour of the colonies; other strains were called bacteroides species.

Antibiotic sensitivity testing was performed on each isolate by the disc diffusion technique using Isosensitest agar (Oxoid) (supplemented with 5\% chocolated horseblood, haemin and menadione when necessary). Escherichia coli NCTC 10418 and Staphylococcus aureus NCTC 6571 were used as control organisms. 


\section{Results}

Eighty-five organisms were isolated from the 12 patients in the study. Fifty-seven $(67 \%)$ organisms were aerobes and $28(33 \%)$ were obligate anaerobes. Both aerobic and anaerobic organisms were isolated from all patients. The mean number of isolates per patient was seven (range 5-10).

The distribution of aerobic and anaerobic organisms isolated in each patient is shown in table 1 . Alpha and non-haemolytic streptococci comprised $49 \%$ of the total aerobic growth. Coagulase negative staphylococci, lactobacilli, and corynebacterium species were frequent isolates. Coliforms were isolated in four patients.

Anaerobic Gram-negative bacilli were isolated from 11 patients. Of these, five were identified as Bacteroides melaninogenicus and two as Bacteroides fragilis. Anaerobic cocci were isolated from six patients.

The corresponding antibiotic sensitivities are shown in table 2. Ninety-one per cent of all strains were sensitive to ampicillin. Only $34 \%$ of aerobic strains were sensitive to gentamicin. All obligate anaerobes were sensitive to metronidazole.

\section{Discussion}

The microbial flora of the oesophagus has attracted little attention. When considering the normal body flora, authors have omitted to mention the organ or merely comment on the transient nature of the flora

Table 1 Distribution of isolates in 12 patients

\begin{tabular}{|c|c|c|c|c|c|c|c|c|c|c|c|c|c|}
\hline \multirow{2}{*}{$\begin{array}{l}\text { Organisms } \\
(+=\text { strain of organism })\end{array}$} & \multicolumn{13}{|c|}{ Patients } \\
\hline & 1 & 2 & 3 & 4 & 5 & 6 & 7 & 8 & 9 & 10 & 11 & 12 & Total strains isolated \\
\hline \multicolumn{14}{|l|}{ Aerobes } \\
\hline a-Haemolytic Streptococcus & .. & - & $+\therefore$ & & ++4 & + & $+\dot{q}$ & & & $\because$ & $\therefore$ &.- & 14 \\
\hline Non-Haemolytic Streptococcus & & 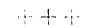 & + & & $\div$ & ++4 & $\div$ & & + & + & - & $\therefore$ & 14 \\
\hline Coagulase - ve Staphylococcus & $\therefore \therefore$ & + & & & & & + & & $-t$ & -+ & $\therefore$ & & 7 \\
\hline Lactobacillus $\mathrm{Sp}$ & + & + & & + & & + & 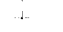 & & - & & $\div$ & & 7 \\
\hline Corynebacterium Sp & & & & + & + & & & & & $\cdot$ & & & 4 \\
\hline Neisseria catarrhalis & & & & & & & & $\rightarrow$ & & +1. & & & 2 \\
\hline E Coli & & & & $\therefore-$ & & & + & & & + & & & 3 \\
\hline Proteus mirabilis & + & & & & & & & & & & & & 1 \\
\hline Branching Gram + ve Rod & & & & & & & + & & & & & & $i$ \\
\hline \multicolumn{14}{|l|}{ Anaerobes } \\
\hline Bacteroides melaninogenicus & & & & & + & & & & + & $\therefore-$ & + & & 5 \\
\hline Bacteroides fragilis & + & & & + & & & & & & & & & 2 \\
\hline Other Bacteroides Sp & & + & & & + & + & $\therefore$ & : & $\therefore$ & & & & 6 \\
\hline Anaerobic cocci & & - & $\therefore$ & & & +-- & & $-!$ & & & $\therefore$ & . & 7 \\
\hline Clostridium $S p$ & & & $\div$ & & + & & & & & . & $\therefore$ & & 5 \\
\hline Other Anaerobes & & + & & & & + & & & & & & & 3 \\
\hline Yeasts & $-\uparrow$ & + & $\therefore-$ & & & & & & & & & & 4 \\
\hline Total strains per patient & 7 & 10 & 6 & 5 & 8 & 9 & 8 & 5 & 7 & 8 & 7 & 5 & \\
\hline
\end{tabular}

Table 2 Antibiotic sensitivity of species (yeast excluded)

\begin{tabular}{|c|c|c|c|c|c|c|c|c|}
\hline \multirow[t]{2}{*}{ Aerobic organisms } & \multirow{2}{*}{$\begin{array}{l}\text { Number } \\
\text { of strains }\end{array}$} & \multicolumn{7}{|c|}{ Antibiotic ( $\%$ strain sensitive) } \\
\hline & & Penicillin & Ampicillin & Tetracycline & Erythromycin & Clindamycin & Gentamicin & Metronidazole \\
\hline${ }_{a}$-Haem Streptococci & 14 & 100 & 100 & 78 & 100 & 100 & 7 & - \\
\hline Non-Haem Streptococci & 14 & 93 & 100 & 71 & 100 & 93 & 0 & $\ldots$ \\
\hline Staphylococci $S p$ & 7 & 43 & 43 & 100 & 100 & 100 & 86 & - \\
\hline Lactobacilli $S p$ & 7 & 100 & 100 & 71 & 100 & 83 & 29 & - \\
\hline Corynebacterium $S p$ & 4 & 100 & 100 & 75 & 75 & 50 & 75 & - \\
\hline Coliforms & 4 & 0 & 75 & 50 & 0 & 0 & 100 & $\cdots .$. \\
\hline Others & 3 & 100 & 100 & 100 & 100 & 100 & 67 & - \\
\hline Aerobic sensitivity total & 53 & 83 & 91 & 77 & 90 & 83 & 34 & - \\
\hline \multicolumn{9}{|l|}{ Anaerobes } \\
\hline Bacteroides $S p$ & 13 & 85 & 85 & 85 & 85 & 100 & 一 & 100 \\
\hline Anaerobic Cocci & 7 & 100 & 100 & 57 & 100 & 100 & $\ldots$ & 100 \\
\hline Others & 8 & 100 & 100 & 75 & 63 & 63 & - & 100 \\
\hline \multirow{3}{*}{$\begin{array}{l}\text { Anaerobic sensitivity total } \\
\text { Combined aerobic } \\
\text { anaerobic sensitivity }\end{array}$} & 28 & 93 & 93 & 75 & 81 & 83 & - & 100 \\
\hline & & & & & & & & \\
\hline & 81 & 86 & 91 & 77 & 83 & 83 & 22 & 35 \\
\hline
\end{tabular}


which reflects the episodic passage of swallowed material. ${ }^{34}$ This study has demonstrated that the dilated oesophagus proximal to a malignant stricture harbours an extensive aerobic and anaerobic flora. Although the number of patients sampled is small, the flora demonstrated in each case is similar and therefore we doubt whether inclusion of further patients would alter the results significantly.

The anaerobes were isolated by carefully employed standard bacteriological techniques. It may be that improved anaerobic laboratory facilities might increase the yield of fastidious strains. Although no formal quantitative analysis was performed, direct plating of material yielded a profuse growth of most organisms.

The spectrum of organisms was similar to that found in the oral cavity suggesting that the flora is primarily of oral origin. ${ }^{5}$ There was no possibility of contamination of specimens by oral secretions or by a nasogastric tube since all the samples were obtained at thoracotomy. It would appear that the proximal oesophagus acts as a reservoir for oral secretions in these patients. The presence of small numbers of E coli, Proteus mirabilis, and Bacteroides fragilis does not preclude the probability of an oral origin since these strains may be isolated from the saliva of both hospitalised patients and those receiving antibiotic therapy. ${ }^{6}$ p

The pathogenicity and stability of this flora remains speculative. The contribution, however, from swallowed saliva to the ecology of this particular microhabitat must be regular and presumably significant. Furthermore specimens were obtained from the proximal oesophageal resection edge, which forms part of the gastro-oesophageal anastomosis and hence these organisms may be available for implantation into the thorax in the event of anastomotic leakage. Pathogenicity of individual members of a flora, when considered in isolation, may not be impressive (lactobacilli, coagulase negative staphylococci, corynebacterium species), but the infectivity of a mixed inocula often exceeds the sum of the contributions of the individual components of the inocula.

It is notable that most of these organisms have been previously isolated as pathogens in thoracic empyemas. The anaerobes may be of particular importance in this respect. Bartlett et al, ${ }^{8}$ in a review of 83 cases of empyema, recovered anaerobic bacteria in $76 \%$. Bacteroides melaninogenicus, previously considered to be an oral commensal, was the second most frequently isolated anaerobic pathogen. Furthermore the spectrum of isolates in Bartlett's study correlates very closely with the flora described above. The reduction in apparently "sterile" empyemas since the introduction of adequate anaerobic culture techniques provides further evidence for the role of anaerobes in thoracic sepsis. ${ }^{9}$

In the present study most aerobic organisms were sensitive to ampicillin. Although gentamicin was effective against only one-third of aerobes, it was highly effective against the small number of ampicillin-resistant coliforms. Metronidazole was effective against all anaerobes. The presence of penicillin-resistant Bacteroides fragilis and the emergence of penicillin-resistant Bacteroides melaninogenicus, ${ }^{10}$ underlines the necessity to include metronidazole in any therapeutic regime directed against the anaerobic component of sepsis associated with oesophageal surgery.

The role of antibiotic prophylaxis in oesophageal surgery has not been clearly defined. In the present study, the most appropriate antibiotic combination for prophylaxis would appear to be ampicillin or penicillin with gentamicin and metronidazole. It is of interest to note that Little et al have demonstrated that prophylactic cephamandole in patients undergoing oesophageal resection for carcinoma significantly reduced the incidence of wound infection. ${ }^{11}$

In the management of an established oesophageal anastomotic leak, surgical elimination of the septic focus, as advocated by Belsey, is of primary importance. ${ }^{12}$ In the absence of specific bacteriological information and until further data regarding the relative pathogenicity of individual components of the flora are available, we would recommend the use of the same combination of ampicillin or penicillin with gentamicin and metronidazole in anastomotic leakage.

\section{References}

${ }^{1}$ Earlham R, Cunha-Melo JR. Oesophageal squamous cell carcinoma. I. A review of surgery. Br J Surg 1980; 67:381-90.

${ }^{2}$ Hermack AS, Crawford DG. The oesophageal anastomotic leak. Am J Surg 1976;132:794-8.

${ }^{3}$ Jawetz E, Melinick JL, Adelberg EA. Review of medical microbiology. Eleventh edition. Los Altos, California: Lange Medical Publications, 1974:256.

${ }^{4}$ Williams REO. Benefit and mischief from commensal bacteria. J Clin Pathol 1973;26:811-8.

${ }^{5}$ Hardie JM, Bowden GH. The normal microbial flora of the mouth. In: Skinner FA, Carr JG, eds. The normal microbial flora of man. London: Academic Press, 1974: 47-83.

6 Percival A, Roberts C. Significance of enterobacteria and pseudomonas in sputum. In: Microbiology of the seventies. A symposium held at Brompton Hospital. London: Butterworths, 1972:154-65.

7 Duerdin BI. The isolation and identification of Bacteroides species from the normal human gingival flora. $J$ Med Microbiol 1980;13:89-101.

${ }^{8}$ Bartlett JG, Gorbach SL, Thadepalli H, Finegold SM. Bacteriology of empyema. Lancet 1974;1:338-40.

${ }^{9}$ Editorial: Anaerobes in pleuropulmonary infections. Lancet 1976;1:289-90. 
${ }^{10}$ Holbrook WP, Duerdin BI, Deacon AG. Classification of Bacteroides melaninogenicus and related species. $J$ Appl Microbiol 1977;42:259-73.

${ }^{11}$ Little G, Alvins E, Matthews HR. Prophylactic antibiotics in oesophageal resection. Thorax $1981 ; 36: 73$.

12 Triggiani E, Belsey R. Oesophageal trauma: incidence, diagnosis and management. Thorax 1977;32:241-9.

Duke Medical Center Postgraduate Course in Diagnostic Imaging 26-30 July 1982, Atlantic Beach, North Carolina. Registration fee $\$ 375$ (US dollars); $\$ 200$ for those in training if accompanied by a letter from the head of department.

This five-day postgraduate meeting will be presented by the faculty of the Department of Radiology, Duke University Medical Center and is designed for radiologists but open to other physicians whether in training or practice. The scientific sessions will cover adult and paediatric diagnostic radiology including ultrasound, CT, and nuclear medicine. For further details please contact Donald R Kirks MD, Department of Radiology, Box 3834, Duke University Medical Center, Durham, NC 27710, USA. 